\title{
Unilateral Global Interval Bifurcation for the Hessian Equation and Its Applications
}

\author{
Wenguo Shen \\ Department of Basic Courses, Lanzhou Institute of Technology, Lanzhou 730050, China \\ Correspondence should be addressed to Wenguo Shen; shenwg369@163.com
}

Received 14 November 2019; Accepted 31 January 2020; Published 13 April 2020

Academic Editor: Antonio Iannizzotto

Copyright (c) 2020 Wenguo Shen. This is an open access article distributed under the Creative Commons Attribution License, which permits unrestricted use, distribution, and reproduction in any medium, provided the original work is properly cited.

In this paper, we establish a unilateral global bifurcation result from the interval for the $k$-Hessian equations with nondifferentiable nonlinearity. By applying the above result, we shall prove the existence of the principal half-eigenvalues for the half quasilinear problems. Furthermore, we shall determine the interval of $\gamma$, in which there exist one-sign solutions for the following $k$-Hessian equations: $\begin{cases}S_{k}\left(D^{2} u\right)=\alpha(x)\left(-u^{+}\right)^{k}+\beta(x)\left(-u^{-}\right)^{k}+\gamma a(x) f(u), & \text { in } B, \\ u(x)=0, & \text { on } \partial B .\end{cases}$

\section{Introduction}

We first consider the following $k$-Hessian equation:

$$
\begin{cases}S_{k}\left(D^{2} u\right)=\lambda a(x)(-u)^{k}+g(x,-u, \lambda), & \text { in } B, \\ u(x)=0, & \text { on } \partial B,\end{cases}
$$

where $D^{2} u=\left(\partial^{2} u / \partial x_{i} \partial x_{j}\right)$ is the Hessian matrix of $u, B$ is the unit ball of $\mathbb{R}^{N}, a(x)$ is a weighted function, $\lambda$ is a positive parameter, and $g \in C\left(\bar{B} \times \mathbb{R}^{2}\right)$. Here, $S_{k}\left(D^{2} u\right)=$ $\sigma_{k}\left(\lambda\left(D^{2} u\right)\right)=\sum_{1 \leq i_{1}<\cdots<i_{k} \leq N} \lambda_{i_{1}} \ldots \lambda_{i_{k}}$ is the $k$-Hessian operator of $u$, where $\lambda\left(D^{2} u\right):=\lambda=\left(\lambda_{1}, \ldots, \lambda_{k}\right)$ is the eigenvalues of $D^{2} u$.
The $k$-Hessian equations are a type of important fully nonlinear equations. Historically, there are a large amount of papers in the literature on the existence, regularity, and the qualitative properties of solutions for the $k$-Hessian equation; see [1-13] and the references therein. Wang [14] has proved the existence of the first eigenvalue $\lambda_{1}$ of problem (1) with $\lambda a(x)=\lambda^{k}$ and $g=0$. When $k=1$, the $k$-Hessian equation is just the semilinear elliptic equation. The $N$ Hessian equation is just the Monge-Ampère equation.

Recently, in [15], the radial solutions of problem (1) is equivalent to the solutions of the following boundary value problem:

$$
\left\{\begin{array}{l}
\left(r^{N-k}\left(u^{\prime}\right)^{k}\right)^{\prime}=\frac{N}{C_{N}^{k}} r^{N-1}\left[\lambda a(r)(-u)^{k}+g(r,-u, \lambda)\right], \quad r \in(0,1), \\
u^{\prime}(0)=u(1)=0
\end{array}\right.
$$

where $r=|x|$ with $x \in B$. By a solution of problem (2), we understand that it is a function which belongs to $C^{2}[0,1]$ and satisfies (2). Dai [15] established a global bifurcation result for the $k$-Hessian equation (2) with $\lambda a(r)(-u)^{k}+$ $g(r,-u, \lambda)$ equal $\lambda^{k}\left((-u)^{k}+g(-u)\right)$.
However, in [15], the nonlinearities are differentiable at the origin. In [16], Berestycki established an important global bifurcation theorem from intervals for a class of second-order problems involving nondifferentiable nonlinearity. Recently, Dai and Ma $[17,18]$ considered 
one-dimensional and high-dimensional $p$-Laplacian problems involving nondifferentiable nonlinearity, respectively. In 2018, Shen $[19,20]$ established interval bifurcation theorems for the Kirchhoff-type problems and the Monge-Ampère equations involving nondifferentiable nonlinearity, respectively.

Motivated by the above papers, in this paper, we shall establish a unilateral global interval bifurcation result for the following $k$-Hessian equations with nondifferentiable nonlinearity:

$$
\begin{cases}S_{k}\left(D^{2} u\right)=\lambda a(x)(-u)^{k}+F(x,-u, \lambda), & \text { in } B, \\ u(x)=0, & \text { on } \partial B,\end{cases}
$$

where $B$ is the unit open ball of $\mathbb{R}^{N}$ and the nonlinear term $F$ has the form $F=f+g$, where $f, g \in C\left(\bar{B} \times \mathbb{R}^{2}\right)$ are radially symmetric with respect to $x$.

It is clear that the radial solutions of (3) is equivalent to the solutions of the following problem:

$$
\left\{\begin{array}{l}
\left(r^{N-k}\left(u^{\prime}\right)^{k}\right)^{\prime}=\frac{N}{C_{N}^{k}} r^{N-1}\left[\lambda a(r)(-u)^{k}+F(r,-u, \lambda)\right], \quad r \in(0,1) \\
u^{\prime}(0)=u(1)=0
\end{array}\right.
$$

where $r=|x|$ with $x \in B$.

On the other hand, half-linear or half-quasilinear problems have attracted the attention of some specialists (see [16-20]). On the above basis of the unilateral global interval bifurcation result, we shall study the following half-quasilinear eigenvalue problem:

$$
\left\{\begin{array}{lr}
S_{k}\left(D^{2} u\right)=\lambda a(x)(-u)^{k}+\alpha(x)\left(-u^{+}\right)^{k}+\beta(x)\left(-u^{-}\right)^{k}, & \text { in } B, \\
u(x)=0, & \text { on } \partial B,
\end{array}\right.
$$

where $u^{+}=\max \{u, 0\}, u^{-}=-\min \{u, 0\}$, and $\alpha(x), \beta(x) \epsilon$ $C(\bar{B})$ are radially symmetric.

It is clear that the radial solutions of (5) is equivalent to the solutions of the following problem:

$$
\left\{\begin{array}{l}
\left(r^{N-k}\left(u^{\prime}\right)^{k}\right)^{\prime}=\frac{N}{C_{N}^{k}} r^{N-1}\left[\lambda a(r)(-u)^{k}+\alpha(r)\left(-u^{+}\right)^{k}+\beta(r)\left(-u^{-}\right)^{k}\right], \quad r \in(0,1), \\
u^{\prime}(0)=u(1)=0 .
\end{array}\right.
$$

Following the above eigenvalue theory (see Theorem 2), we shall investigate the existence of radial one-sign solutions for the following problem:
It is clear that the radial solutions of (7) is equivalent to the solutions of the following problem:

$$
\begin{cases}S_{k}\left(D^{2} u\right)=\alpha(x)\left(-u^{+}\right)^{k}+\beta(x)\left(-u^{-}\right)^{k}+\lambda a(x) f(u) & \text { in } B, \\ u(x)=0, & \text { on } \partial B .\end{cases}
$$

$$
\left\{\begin{array}{l}
\left(r^{N-k}\left(u^{\prime}\right)^{k}\right)^{\prime}=\frac{N}{C_{N}^{k}} r^{N-1}\left[\alpha(r)\left(-u^{+}\right)^{k}+\beta(r)\left(-u^{-}\right)^{k}+\gamma a(r) f(u)\right], \quad r \in(0,1), \\
u^{\prime}(0)=u(1)=0 .
\end{array}\right.
$$

The rest of this paper is arranged as follows. In Section 2, we give some preliminaries. In Section 3, we establish the unilateral global interval bifurcation result for $k$-Hessian equation (4) (see Theorem 1). In Section 4, on the basis of the unilateral global interval bifurcation result, we shall prove the existence of the principal eigenvalues for $k$-Hessian equation (6) (see Theorem 2). In Section 5, we shall investigate the existence of one-sign solutions for $k$-Hessian equation (8). 


\section{Preliminaries}

Let $Y=C[0,1]$ with the norm $\|u\|_{\infty}=\max _{r \in[0,1]}|u(r)|$. Let $E:=\left\{u(r) \in C^{1}(0,1) \mid u^{\prime}(0)=u(1)=0\right\}$ with the norm $\|u\|=\max \left\{\|u\|_{\infty},\left\|u^{\prime}\right\|_{\infty}\right\}$. Let $P^{+}=\{u \in E: u(r)>0, r \in$ $(0,1)\}$ and $P^{-}=-P^{+}, P=P^{+} \cup P^{-}$. Let $K^{+}=\mathbb{R} \times P^{+}$and $K=\mathbb{R} \times P$ under the product topology.

By Sections 5 and 6 in [15], we firstly consider the following problem:

$$
\left\{\begin{array}{l}
\left(r^{N-k}\left(-\nu^{\prime}\right)^{k}\right)^{\prime}=h(r), r \in(0,1), \\
\nu^{\prime}(0)=v(1)=0
\end{array}\right.
$$

Let us define the operator $G_{k}(h): E \longrightarrow E$ by

$$
G_{k}(h)=v(r)=\int_{1}^{r}\left(s^{k-N} \int_{s}^{0} h(\tau) \mathrm{d} \tau\right)^{1 / k} \mathrm{~d} s, \quad 0 \leq r \leq 1 .
$$

For a given $h \in Y, G_{k}(h): Y \longrightarrow E$ is a completely continuous and (10) is equivalent to (9).

With a simple transformation $v=-u$, problem (2) can be equivalently written as

$$
\left\{\begin{array}{l}
\left(r^{N-k}\left(-\nu^{\prime}\right)^{k}\right)^{\prime}=\frac{N}{C_{N}^{k}} r^{N-1}\left[\lambda a(r) v^{k}+g(r, v, \lambda)\right], \quad r \in(0,1), \\
\nu^{\prime}(0)=\nu(1)=0,
\end{array}\right.
$$

where $g \in C\left([0,1] \times\left(\mathbb{R}^{+}\right)^{2}\right)$ and $a$ satisfies the following conditions:

(A0) $a(x) \in C(\bar{B})$ is radially symmetric and $a(r) \geq 0$, $a(r) \neq \equiv$ on any subinterval of $[0,1]$, where $r=|x|$ with $x \in \bar{B}$

(A1) $g(r, s, \lambda)=o\left(s^{k}\right)$ near $s=0$ uniformly in $r \in(0,1)$ and $\lambda$ on bounded sets

And $g$ also satisfies the following subcritical growth restriction (see [15], p.4-(1.5)):

$$
|g(r, s, \lambda)| \leq C\left(1+|s|^{q-1}\right),
$$

uniformly for $r \in(0,1), \lambda$ on bounded sets, and some $q \in\left(0, k^{*}\right)$. Here,

$$
k^{*}= \begin{cases}\frac{N(k+1)}{N-2 k}, & \text { if } k<\frac{N}{2}, \\ +\infty, & \text { if } k \geq \frac{N}{2}\end{cases}
$$

is the critical exponent for the $k$-Hessian operator, which was first determined in [21].

Define the Nemytskii operator $H: \mathbb{R} \times E \longrightarrow Y$ by

$$
H(\mu, v)(r):=\frac{N}{C_{N}^{k}} r^{N-1}\left[\mu a(r) v^{k}+g(r, v, \mu)\right] .
$$

Then, it is clear that $H$ is a continuous (compact) operator and problem (11) can be equivalently written as

$$
v=G_{k}[H(\mu, v)]:=F(\mu, v),
$$

where $F: \mathbb{R} \times E \longrightarrow E$ is a completely continuous and $F(\mu, 0)=0, \forall \mu \in \mathbb{R}$.

Now, we consider the following eigenvalue problem:

$$
\left\{\begin{array}{l}
\left(r^{N-k}\left(-v^{\prime}\right)^{k}\right)^{\prime}=\lambda \frac{N}{C_{N}^{k}} r^{N-1} a(r) v^{k}, \quad r \in(0,1), \\
v^{\prime}(0)=v(1)=0 .
\end{array}\right.
$$

By the same proof as in Theorem 4.1 of [14], we can show that problem (16) possesses the first eigenvalue $\lambda_{1}$ which is positive, simple, unique and the corresponding eigenfunctions are positive in $(0,1)$.

By Rabinowitz [22], using the same method to prove ([15], Theorem 1.1) with obvious changes, we may get the following global bifurcation result.

Lemma 1 (see [15], Theorem 1.1). Assume that (A0), (A1), and (12) hold. Then, $\left(\lambda_{1}, 0\right)$ is the unique bifurcation point of problem (11) and there exists an unbounded continuum $C \subseteq\left(K \cup\left\{\left(\lambda_{1}, 0\right)\right\}\right)$ of solutions to problem (11) emanating from $\left(\lambda_{1}, 0\right)$.

By Dancer ([23], Theorem 2), under the conditions (A0), (A1), and (12), one can obtain that problem (11) has two distinct unbounded subcontinua $C^{+}$and $C^{-}$, consisting of the bifurcation branch $C$ emanating from $\left(\lambda_{1}, 0\right)$, which satisfy:

Lemma 2 (see ([23], Theorem 2)). Both $C^{+}$and $C^{-}$are unbounded and

$$
C^{\nu} \subset\left(\left(\mathbb{R} \times P^{\nu}\right) \cup\left\{\left(\lambda_{1}, 0\right)\right\}\right),
$$

where $v \in\{+,-\}$.

Next, we give the following results.

Lemma 3. Let $I$ be an interval and if $y$ and $z$ are functions such that $y, z, \varphi_{k}\left(y^{\prime}\right)$ and $\varphi_{k}\left(z^{\prime}\right)$ are differentiable on I and 
$y(t)>0, z(t)>0, y^{\prime}(t)<0, z^{\prime}(t)<0$ for $t \in I$. Then, we have the following identity:

$$
\begin{aligned}
\frac{\mathrm{d}}{\mathrm{d} t}\left\{\frac{y}{\varphi_{k}(z)}\left[\varphi_{k}(y) \varphi_{k}\left(-z^{\prime}\right)-\varphi_{k}(z) \varphi_{k}\left(-y^{\prime}\right)\right]\right\} \\
=\frac{y}{\varphi_{k}(z)}\left[\varphi_{k}(y) L_{k}[z]-\varphi_{k}(z) L_{k}[y]\right] \\
\quad+r^{N-k}\left[\left(-y^{\prime}\right)^{k+1}+k\left(\frac{-y z^{\prime}}{z}\right)^{k+1}+(k+1) y^{k} y^{\prime}\left(\frac{-z^{\prime}}{z}\right)^{k}\right],
\end{aligned}
$$

where $\varphi_{k}(s)=r^{N-k} s^{k}, L_{k}[y]=\left(\varphi_{k}\left(-y^{\prime}\right)\right)^{\prime}$.

Proof. Similar the proof of Lemma 2.3 of [15] with obvious change, the left-hand side of (18) equals

$$
\begin{aligned}
\frac{\mathrm{d}}{\mathrm{d} t}\{ & \left.\frac{y^{k+1} r^{N-k}\left(-z^{\prime}\right)^{k}}{z^{k}}-y r^{N-k}\left(-y^{\prime}\right)^{k}\right\} \\
= & \frac{\left[(k+1) y^{k} y^{\prime} r^{N-k}\left(-z^{\prime}\right)^{k}+y^{k+1}\left(r^{N-k}\left(-z^{\prime}\right)^{k}\right)^{\prime}\right] z^{k}-y^{k+1} r^{N-k}\left(-z^{\prime}\right)^{k} k z^{k-1} z^{\prime}}{z^{2 k}} \\
& -y^{\prime} r^{N-k}\left(-y^{\prime}\right)^{k}-y\left(r^{N-k}\left(-y^{\prime}\right)^{k}\right)^{\prime}=\frac{y}{\varphi_{k}(z)}\left[\varphi_{k}(y) L_{k}[z]-\varphi_{k}(z) L_{k}[y]\right] \\
& +r^{N-k}\left[\left(-y^{\prime}\right)^{k+1}+k\left(\frac{-y z^{\prime}}{z}\right)^{k+1}+(k+1) y^{k} y^{\prime}\left(\frac{-z^{\prime}}{z}\right)^{k}\right] .
\end{aligned}
$$

Lemma 4. In (18) of Lemma 3, by Young's inequality, we get

$$
\left[\left(-y^{\prime}\right)^{k+1}+k\left(\frac{-y z^{\prime}}{z}\right)^{k+1}+(k+1) y^{k} y^{\prime}\left(\frac{-z^{\prime}}{z}\right)^{k}\right] \geq 0 \text {, }
$$

and the equality holds if and only if sgny =sgnz and $\left|y^{\prime} / y\right|^{k+1}=\left|z^{\prime} / z\right|^{k+1}$.

Proof. Now recall Young's inequality:

$$
A B \leq \frac{A^{\alpha}}{\alpha}+\frac{B^{\beta}}{\beta}
$$

where $A, B \in \mathbb{R}^{+}, \alpha, \beta>1,1 / \alpha+1 / \beta=1$.

Let $\quad \alpha=k+1, \beta=(k+1) / k, \quad A=-(k+1)^{1 /(k+1)} y^{\prime}$, and $B=(k+1)^{k /(k+1)} y^{k}\left(-z^{\prime} / z\right)^{k}$ in (21). We obtain inequality (20) holds. sult:

By Lemma 3 and Lemma 4, we have the following re-

Lemma 5. In (18) of Lemma 3, we have

$$
\int_{0}^{1} \frac{y}{\varphi_{k}(z)}\left(\varphi_{k}(y) L_{k}[z]-\varphi_{k}(z) L_{k}[y]\right) \mathrm{d} r \leq 0 .
$$

Proof. By (18) of Lemma 3, it follows that

$$
\begin{aligned}
& \int_{0}^{1}\left\{\frac{y}{\varphi_{k}(z)}\left[\varphi_{k}(y) \varphi_{k}\left(-z^{\prime}\right)-\varphi_{k}(z) \varphi_{k}\left(-y^{\prime}\right)\right]\right\}^{\prime} \mathrm{d} r \\
& =\int_{0}^{1} r^{N-k}\left[\left(-y^{\prime}\right)^{k+1}+k\left(\frac{-y z^{\prime}}{z}\right)^{k+1}+(k+1) y^{k} y^{\prime}\left(\frac{-z^{\prime}}{z}\right)^{k}\right] \mathrm{d} r \\
& \quad+\int_{0}^{1} \frac{y}{\varphi_{k}(z)}\left[\varphi_{k}(y) L_{k}[z]-\varphi_{k}(z) L_{k}[y]\right] \mathrm{d} r .
\end{aligned}
$$

Similar to the proof as (2.3) in Lemma 2.3 of [15], we can show that the left-hand side of (23) equals 0 . By Lemma 4 , we have the result.

In order to treat the problems with nonasymptotic nonlinearity at 0 and $\infty$, we use Whyburn-type superior limit theorems. However, if the collection of the infinite sequence of sets is unbounded, Whyburn's limit theorem ([24], Theorem 9.1) cannot be used directly because the collection may not be relatively compact. In order to overcome this difficulty, Dai [25] established the following results.

Lemma 6 (see [25], Lemma 2.5). Let $X$ be a normal space and let $\left\{C_{n} \mid n=1,2, \ldots\right\}$ be a sequence of unbounded connected subsets of $X$. Assume that

(i) There exists $z^{*} \in \liminf _{n \longrightarrow+\infty} C_{n}$ with $\left\|z^{*}\right\|<+\infty$. 
(ii) For every $R>0,\left(\cup_{n=1}^{+\infty} C_{n}\right) \cap \bar{B}_{R}$ is a relatively compact set of $X$, where

$$
B_{R}=\{x \in X \mid\|x\| \leq R\}
$$

Then, $\mathbb{D}:=\lim \sup _{n \rightarrow \infty} C_{n}$ is unbounded, closed, and connected.

Lemma 7 (see [25], Theorem 1.2). Let $X$ be a normal vector space and let $\left\{C_{n} \mid n=1,2, \ldots\right\}$ be a sequence of unbounded connected subsets of $X$. Assume that

(i) There exists $z^{*} \in \liminf _{n \longrightarrow+\infty} C_{n}$ with $\left\|z^{*}\right\|=+\infty$.

(ii) There exists a homeomorphism $T: X \longrightarrow X$ such that $\left\|T\left(z^{*}\right)\right\|<+\infty$ and $\left\{T\left(C_{n}\right)\right\}$ be a sequence of unbounded connected subsets in $X$.

(iii) For every $R>0,\left(\cup_{n=1}^{+\infty} C_{n}\right) \cap \bar{B}_{R}$ is a relatively compact set of $X$, where

$$
B_{R}=\{x \in X \mid\|x\| \leq R\}
$$

Then, $\mathbb{D}:=\lim \sup _{n \rightarrow \infty} C_{n}$ is unbounded, closed, and connected.

Lemma 8 (see [25], Corollary 2.3). Let $(X, \rho)$ be a metric space. If $\left\{C_{i}\right\}_{i \in \mathbb{N}}$ is a sequence of sets whose limit superior is $L$ and there exists a homeomorphism $T: X \longrightarrow X$ such that for every $R>0, \quad\left(\cup_{i=1}^{+\infty} T\left(C_{i}\right)\right) \cap \bar{B}_{R}$ is a relatively compact set; then, for each $\epsilon>0$, there exists an $m$ such that for every $n>m, C_{n} \subset V_{\epsilon}(L)$, where $V_{\epsilon}(L)$ denotes the set of all points $p$ with $\rho(p, x)<\epsilon$ for any $x \in L$.

\section{Global Bifurcation from an Interval}

With a simple transformation $v=-u$, problem (4) can be equivalently written as

$$
\left\{\begin{array}{l}
\left(r^{N-k}\left(-v^{\prime}\right)^{k}\right)^{\prime}=\frac{N}{C_{N}^{k}} r^{N-1}\left[\lambda a(r) v^{k}+F(r, v, \lambda)\right], \quad r \in(0,1), \\
v^{\prime}(0)=v(1)=0,
\end{array}\right.
$$

where $a$ satisfying (A0), $F=f+g, f, g \in C\left([0,1] \times\left(\mathbb{R}^{+}\right)^{2}\right)$, $g$ satisfying (A1) and (12), and $f$ satisfying

(A2) $\left|f(r, s, \lambda) / s^{k}\right| \leq M$, for all $r \in(0,1), 0<s \leq 1$, and all $\lambda \in \mathbb{R}$, where $M$ is a positive constant.

Let $S^{v}$ denote the closure in $\mathbb{R} \times E$ of the set of nontrivial solutions $(\lambda, v)$ of $(26)$ with $v \in P^{v}$. By an argument similar to that of ([15], Lemma 5.1) with obvious changes, we can show that the following existence and uniqueness theorem is valid for problem (26).

Lemma 9 (see [15], Lemma 5.1). If $(\lambda, v)$ is a solution of (26) under the assumptions of (A0), (A1), (12), and (A2), and $v$ has a double zero, then $v \equiv 0$.

The first main result for (26) is the following theorem.

Theorem 1. Let (A0), (A1), (12), and (A2) hold. Let $d=M / a_{0}$, where $a_{0}=\min _{r \in[0,1]} a(r)$, and let $I=\left[\lambda_{1}-d\right.$, $\left.\lambda_{1}+d\right]$. The component $C^{\nu}$ of $S^{\nu} \cup(I \times\{0\})$, containing $I \times\{0\}$, is unbounded and lies in $K^{\nu} \cup(I \times\{0\})$ for $v \in\{+,-\}$.

To prove Theorem 1, we introduce the following auxiliary approximate problem:

$$
\left\{\begin{array}{l}
\left(r^{N-k}\left(-v^{\prime}\right)^{k}\right)^{\prime}=\frac{N}{C_{N}^{k}} r^{N-1}\left[\lambda a(r) v^{k}+f\left(r, v|v|^{\epsilon}, \lambda\right)+g(r, v, \lambda)\right], \quad r \in(0,1), \\
v^{\prime}(0)=v(1)=0 .
\end{array}\right.
$$

To prove Theorem 1, the following lemma will play a key role.

Lemma 10. Let $\epsilon_{n}, 0<\epsilon_{n}<1$, be a sequence converging to 0 . If there exists a sequence $\left(\lambda_{n}, v_{n}\right) \in K^{v}$ such that it is a nontrivial solution of problem (27) corresponding to $\epsilon=\epsilon_{n}$ and $\left(\lambda_{n}, v_{n}\right)$ converges to $(\lambda, 0)$ in $\mathbb{R} \times E$, then $\lambda \in I$.

Proof. Let $w_{n}=v_{n} /\left\|v_{n}\right\|$, then $w_{n}$ satisfies the following problem:

$$
\left\{\begin{array}{l}
\left(r^{N-k}\left(-w_{n}^{\prime}\right)^{k}\right)^{\prime}=\frac{N}{C_{N}^{k}} r^{N-1}\left[\lambda a(r) w_{n}^{k}+\frac{f\left(r, v_{n}\left|v_{n}\right|^{\epsilon}, \lambda_{n}\right)}{\left\|v_{n}\right\|^{k}}+\frac{g\left(r, v_{n}, \lambda_{n}\right)}{\left\|v_{n}\right\|^{k}}\right], \quad r \in(0,1), \\
w_{n}^{\prime}(0)=w_{n}(1)=0 .
\end{array}\right.
$$


Let

$$
\bar{g}(r, v, \lambda)=\max _{0 \leq|s| \leq v}|g(r, s, \lambda)|, \quad \text { for all } r \in(0,1) \text { and } \lambda \text { on bounded sets, }
$$

then, $\bar{g}$ is nondecreasing with respect to $v$ and

$$
\lim _{v \longrightarrow 0^{+}} \frac{\bar{g}(r, v, \lambda)}{v^{k}}=0,
$$

uniformly for $r \in(0,1)$ and $\lambda$ on bounded sets. Furthermore, it follows from (30) that

$$
\frac{|g(r, v, \lambda)|}{\|v\|^{k}} \leq \frac{\bar{g}(r,|v|, \lambda)}{\|v\|^{k}} \leq \frac{\bar{g}\left(r,\|v\|_{\infty}, \lambda\right)}{\|v\|^{k}} \leq \frac{\bar{g}(r,\|v\|, \lambda)}{\|v\|^{k}} \longrightarrow 0, \quad \text { as }\|v\| \longrightarrow 0,
$$

uniformly for $r \in(0,1)$ and $\lambda$ on bounded sets.

Clearly, (A2) implies that

$$
\begin{aligned}
\frac{\left|f\left(r, v_{n}\left|v_{n}\right|^{\varepsilon_{n}}, \lambda_{n}\right)\right|}{\left\|v_{n}\right\|^{k}} & =\left|\frac{f\left(t, v_{n}\left|v_{n}\right|^{\varepsilon_{n}}, \lambda_{n}\right)}{v_{n}^{k}\left|v_{n}\right|^{k \varepsilon_{n}}}\right| \cdot \frac{\left|v_{n}^{k}\right|\left|v_{n}\right|^{k \varepsilon_{n}}}{\left\|v_{n}\right\|^{k}} \\
& \leq M_{1} \cdot\left|v_{n}\right|^{k \varepsilon_{n}} \longrightarrow M,
\end{aligned}
$$

for all $r \in(0,1)$.

Note that $\left\|w_{n}\right\|=1$ implies $\left\|w_{n}\right\|_{\infty} \leq 1$. Using this fact with (31) and (32), we have that $\lambda_{n} N r^{N-1} a(r) w_{n}^{k}+N r^{N-1}$ $f\left(r, v_{n}\left|v_{n}\right|^{\epsilon_{n}}, \lambda_{n}\right) /\left\|v_{n}\right\|^{k}+N r^{N-1} g\left(r, v_{n}, \lambda_{n}\right) /\left\|v_{n}\right\|^{k}$ is bounded in $E$ for $n$ large enough. The compactness of $G_{k}$ implies that $w_{n}$ is converges in $E$. Without loss of generality, we may assume that $w_{n} \longrightarrow w$ in $E$ with $\|w\|=1$. Clearly, we have $w \in \overline{P^{v}}$.

We claim that $w \in P^{v}$. On the contrary, suppose that $w \in \partial P^{v}$, by Lemma 9 , then, $w \equiv 0$, which is a contradiction with $\|w\|=1$.

Now, we deduce the boundedness of $\lambda$. Let $\psi$ be an eigenfunction of problem (16) corresponding to $\lambda_{1}$.

We know that $w_{n}$ satisfies $\left(r^{N-k}\left(-w_{n}^{\prime}\right)^{k}\right)^{\prime}=\left(N / C_{N}^{k}\right) r^{N-1}$ $\left[\lambda a(r) w_{n}^{k}+f\left(r, v_{n}\left|v_{n}\right|^{\varepsilon_{n}}, \lambda_{n}\right) /\left\|v_{n}\right\|^{k}+g\left(r, v_{n}, \lambda_{n}\right) /\left\|v_{n}\right\|^{k}\right], \quad r \in$
$(0,1), w_{n}^{\prime}(0)=w_{n}(1)=0$, and $\psi$ satisfies $\left(r^{N-k}\left(-\psi^{\prime}\right)^{k}\right)^{\prime}=$ $\left(N / C_{N}^{k}\right) r^{N-1} \lambda_{1} a(r) \psi^{k}, r \in(0,1), \psi^{\prime}(0)=\psi(1)=0$.

We can assume without loss of generality that $v=+$. By Lemma 5, it follows that

$$
\begin{aligned}
\int_{0}^{1} \frac{w_{n}}{\varphi_{k}(\psi)}\left(\varphi_{k}\left(w_{n}\right) L[\psi]-\varphi_{k}(\psi) L\left[w_{n}\right]\right) \mathrm{d} r \\
=\int_{0}^{1}\left[\left(\lambda_{1}-\lambda_{n}\right) a(r)-\frac{f\left(r, v_{n}\left|v_{n}\right|^{\varepsilon_{n}}, \lambda_{n}\right)}{\left\|v_{n}\right\|^{k} w_{n}^{k}}-\frac{g\left(r, v_{n}, \lambda_{n}\right)}{\left\|v_{n}\right\|^{N} w_{n}^{k}}\right] \\
\quad \cdot \frac{N}{C_{N}^{k}} r^{N-1} w_{n}^{k+1} \mathrm{~d} r \leq 0 .
\end{aligned}
$$

Similarly, we can also show that

$$
\begin{aligned}
\int_{0}^{1}\left[\left(\lambda_{n}-\lambda_{1}\right) a(r)+\frac{f\left(r, v_{n}\left|v_{n}\right|^{\varepsilon_{n}}, \lambda_{n}\right)}{\left\|v_{n}\right\|^{k} w_{n}^{k}}+\frac{g\left(r, v_{n}, \lambda_{n}\right)}{\left\|v_{n}\right\|^{k} w_{n}^{k}}\right] \\
\cdot \frac{N}{C_{N}^{k}} r^{N-1} \psi^{k+1} \mathrm{~d} r \leq 0 .
\end{aligned}
$$

If $\lambda \leq \lambda_{1}$, considering (33), (A1), and (A2), we have that

$$
\begin{aligned}
\int_{0}^{1}\left(\lambda_{1}-\lambda\right) a(r) \frac{N}{C_{N}^{k}} r^{N-1} w^{k+1} \mathrm{~d} r & \leq \lim _{n \longrightarrow \infty} \int_{0}^{1} \frac{f\left(r, v_{n}\left|v_{n}\right|^{\epsilon_{n}}, \lambda_{n}\right)}{\left\|v_{n}\right\|^{k} w_{n}^{k}} \frac{N}{C_{N}^{k}} r^{N-1} w_{n}^{k+1} \mathrm{~d} r \\
& \leq \lim _{n \longrightarrow \infty} \int_{0}^{1} \frac{f\left(r, v_{n}\left|v_{n}\right|^{\epsilon_{n}}, \lambda_{n}\right)}{v_{n}^{k}\left|v_{n}\right|^{k \epsilon_{n}}}\left|v_{n}\right|^{k \epsilon_{n}} \frac{N}{C_{N}^{k}} r^{N-1} w_{n}^{k+1} \mathrm{~d} r \\
& \leq \int_{0}^{1} M \frac{N}{C_{N}^{k}} r^{N-1} w^{k+1} \mathrm{~d} r .
\end{aligned}
$$


Hence, we get that

$$
\int_{0}^{1}\left(\lambda_{1}-\lambda\right) a_{0} \frac{N}{C_{N}^{k}} r^{N-1} w^{k+1} \mathrm{~d} r \leq \int_{0}^{1} M \frac{N}{C_{N}^{k}} r^{N-1} w^{k+1} \mathrm{~d} r,
$$

which implies $\lambda \geq \lambda_{1}-d$.

If $\lambda \geq \lambda_{1}$, considering (34), (A1), and (A2), we have that

$$
\begin{aligned}
\int_{0}^{1}\left(\lambda-\lambda_{1}\right) a(r) \frac{N}{C_{N}^{k}} r^{N-1} \psi^{k+1} \mathrm{~d} r & \leq \lim _{n \longrightarrow \infty} \int_{0}^{1} \frac{-f\left(r, v_{n}\left|v_{n}\right|^{\varepsilon_{n}}, \lambda_{n}\right)}{\left\|v_{n}\right\|^{k} w_{n}^{k}} \frac{N}{C_{N}^{k}} r^{N-1} \psi^{k+1} \mathrm{~d} r \\
& \leq \lim _{n \longrightarrow \infty} \int_{0}^{1} \frac{-f\left(r, v_{n}\left|v_{n}\right|^{\varepsilon_{n}}, \lambda_{n}\right)}{v_{n}^{k}\left|v_{n}\right|^{k \varepsilon_{n}}}\left|v_{n}\right|^{k \varepsilon_{n}} \frac{N}{C_{N}^{k}} r^{N-1} \psi^{k+1} \mathrm{~d} r \\
& \leq \int_{0}^{1} M \frac{N}{C_{N}^{k}} r^{N-1} \psi^{k+1} \mathrm{~d} r .
\end{aligned}
$$

Hence, we get that

$$
\int_{0}^{1}\left(\lambda-\lambda_{1}\right) a_{0} \frac{N}{C_{N}^{k}} r^{N-1} \psi^{k+1} \mathrm{~d} r \leq \int_{0}^{1} M \frac{N}{C_{N}^{k}} r^{N-1} \psi^{k+1} \mathrm{~d} r,
$$

which implies $\lambda \leq \lambda_{1}-d$.

Therefore, we have that $\lambda \in I$.

Proof of Theorem 1. We only prove the case of $v=+$ since the proof of the case $v=-$ can be given similarly. Let $C^{+}$be the component of $S^{+} \cup(I \times\{0\})$.

First, using the similar method to prove ([17], Theorem 1.1) with obvious changes, we may prove that $C^{+} \subset\left(K^{+} \cup\right.$ $(I \times\{0\}))$.

In the following, we prove that $C^{+}$is unbounded. Suppose on the contrary that $C^{+}$is bounded. Using the similar method to prove Theorem 1 in [16] with obvious changes, we can find a neighborhood $\mathscr{O}$ of $\mathscr{C}^{+}$such that $\partial \odot \cap \mathscr{P}^{+}=\varnothing$.

In order to complete the proof of this theorem, we consider problem (27). For $\epsilon>0$, it is easy to show that nonlinear term $f\left(r, v|v|^{\epsilon}, \lambda\right)+g(r, v, \lambda)$ satisfies the condition (H2). Let

$$
\mathscr{P}_{\epsilon}=\overline{\{(\lambda, v):(\lambda, v) \text { satisfies }(27) \text { and } v \neq 00}^{\mathbb{R} \times E} \text {. }
$$

By Lemma 1, there exists an unbounded continuum $\mathrm{C}_{\epsilon}$ of $\mathrm{S}_{\epsilon}$ bifurcating from $\left(\lambda_{1}, 0\right)$ such that $\mathrm{C}_{\epsilon} \subset\left(K^{+} \cup\left\{\left(\lambda_{1}, 0\right)\right\}\right)$. Furthermore, by Lemma 2, there are two unbounded continua $\mathrm{C}_{\epsilon}^{+}$and $\mathrm{C}_{\epsilon}^{-}$of $\mathrm{S}_{\epsilon}$ bifurcating from $\left(\lambda_{1}, 0\right)$, which are both unbounded and $\mathrm{C}_{\epsilon}^{v} \subset\left(\mathbb{R} \times P^{\nu}\right) \cup\left\{\left(\lambda_{1}, 0\right)\right\}$.
So there exists $\left(\lambda_{\epsilon}, v_{\epsilon}\right) \in \mathscr{C}_{\epsilon}^{+} \cap \partial \mathcal{O}$ for all $\epsilon>0$. Since $a$ is bounded in $K^{+}$, equation (27) shows that $\left(\lambda_{\epsilon}, v_{\epsilon}\right)$ is bounded in $\mathbb{R} \times C^{2}$ independently of $\epsilon$. By the compactness of $G_{k}$, one can find a sequence $\epsilon_{n} \longrightarrow 0$ such that $\left(\lambda_{\epsilon_{n}}, v_{\epsilon_{n}}\right)$ converges to a solution $(\lambda, v)$ of (27). So $v \in \overline{P^{+}}$. If $v \in \partial P^{+}$, then from Lemma 9, it follows that $v \equiv 0$. By Lemma $10, \lambda \in I$, which contradicts the definition of $\mathcal{O}$. On the other hand, if $v \in P^{+}$, then $(\lambda, v) \in \mathscr{P}^{+} \cap \partial \mathcal{O}$ which contradicts $\mathscr{P}^{+} \cap \partial \mathcal{O}=\varnothing$.

From Theorem 1 and its proof, we can easily get the following corollaries.

Corollary 1. There exist two unbounded subcontinua $\mathrm{D}^{+}$and $\mathrm{D}^{-}$of solutions of (26) in $\mathbb{R} \times Y$, bifurcating from $I \times\{0\}$ and $\mathrm{D}^{\nu} \subset\left(\mathbb{R} \times P^{v}\right) \cup(I \times\{0\})$ for $v=+$ and $\nu=-$.

We relax the assumption of $a(x)$ as the following.

(B0) $a(x) \in C(\bar{B})$ is a radially symmetric sign-changing weight.

Corollary 2. Let $(A 3)$ hold and $f \equiv 0$. Then, there exist two unbounded subcontinua $\mathscr{C}^{+}$and $\mathscr{C}^{-}$of solutions of (26) in $\mathbb{R} \times Y$, bifurcating from $(I \times\{0\})$, and $\mathscr{C}^{\nu} \subset\left(\mathbb{R} \times P^{v}\right) \cup(I \times$ $\{0\})$ for $v=+$ and $v=-$.

\section{Spectrum of Half-Quasilinear Eigenvalue Problems}

With a simple transformation $v=-u$, problem (7) can be equivalently written as

$$
\left\{\begin{array}{l}
\left(r^{N-k}\left(-v^{\prime}\right)^{k}\right)^{\prime}=\frac{N}{C_{N}^{k}} r^{N-1}\left[\lambda a(r) v^{k}+\alpha(r)\left(v^{+}\right)^{k}+\beta(r)\left(v^{-}\right)^{k}\right], \quad r \in(0,1), \\
v^{\prime}(0)=v(1)=0
\end{array}\right.
$$


where $v^{+}=\max \{v, 0\}, v^{-}=-\min \{v, 0\}$, and $\alpha(r)$ and $\beta(r)$ satisfy the following:

(A3) $\alpha(r), \beta(r) \in C[0,1]$ are radially symmetric.

Problem (40) is called half quasi-linear because it is positive $k$ homogeneous in the cones $u>0$ and $u<0$. Similar to that of [16], we say that $\lambda$ is a half-eigenvalue of problem (40), if there exists a nontrivial solution $\left(\lambda, u_{\lambda}\right) . \lambda$ is also said to be simple if $v=c u_{\lambda}, c>0$ for all solutions $(\lambda, v)$ of problem (40). A half-eigenvalue is called the principal halfeigenvalue if the corresponding eigenfunction is positive or negative.

By an argument similar to that of $[17,18]$, we can obtain the following theorem.
Theorem 2. There exist two simple half-eigenvalues $\lambda^{+}$and $\lambda^{-}$for problem (40). The corresponding half-linear solutions are in $\left\{\lambda^{+}\right\} \times P^{+}$and $\left\{\lambda^{-}\right\} \times P^{-}$. Furthermore, aside from $\lambda^{+}$ and $\lambda^{-}$, there is no other half-eigenvalue with positive or negative eigenfunction.

Proof. Applying the similar method to prove Theorem 1.2 in [17] or Theorem 3.1 in [18] with obvious changes, by Theorem 1 and Lemma 3-5, we can prove the conclusion.

Naturally, we can consider the bifurcation structure of the perturbation of problem (40) of the following form:

$$
\left\{\begin{array}{l}
\left(r^{N-k}\left(-v^{\prime}\right)^{k}\right)^{\prime}=\frac{N}{C_{N}^{k}} r^{N-1}\left[\lambda a(r) v^{k}+\alpha(r)\left(v^{+}\right)^{k}+\beta(r)\left(v^{-}\right)^{k}+g(r, v, \lambda)\right], \quad r \in(0,1), \\
v^{\prime}(0)=v(1)=0,
\end{array}\right.
$$

where $g$ satisfies (A1) and (12).

Remark 1. By some simple computation, we can show that if $\beta \equiv 0$, then $\lambda^{-}=\lambda_{1}, \alpha \equiv 0$ implies $\lambda^{+}=\lambda_{1}$, and $\alpha=\beta \equiv 0$ implies $\lambda^{+}=\lambda^{-}=\lambda_{1}$.

Remark 2. Note that $\min \left\{\lambda^{+}, \lambda^{-}\right\}$is the least half-eigenvalue for (40).

Using a similar method to prove Theorem 3.1 in [17] or Theorem 3.2 in [18] with obvious changes, by Corollary 1, we may obtain the following result.
Theorem 3. For $v=+,-,\left(\lambda^{v}, 0\right)$ is a bifurcation point for problem (41). Moreover, there exists an unbounded continuum $\mathrm{D}^{v}$ of solutions of problem (41), such that $\mathrm{D}^{v} \subset((\mathbb{R} \times$ $\left.\left.P^{\nu}\right) \cup\left\{\left(\lambda^{\nu}, 0\right)\right\}\right)$.

Remark 3. Theorem 3 indicates that the bifurcation interval $I=\left\{\lambda^{+}, \lambda^{-}\right\}$, i.e., for problem (41), the bifurcation interval $I$ is a finite point set.

\section{One-Sign Solutions for the Hessian Equation}

With a simple transformation $v=-u$, problem (8) can be equivalently written as

$$
\left\{\begin{array}{l}
\left(r^{N-k}\left(-v^{\prime}\right)^{k}\right)^{\prime}=\frac{N}{C_{N}^{k}} r^{N-1}\left[\alpha(r)\left(v^{+}\right)^{k}+\beta(r)\left(v^{-}\right)^{k}+\gamma a(r) f(v)\right], \quad r \in(0,1), \\
v^{\prime}(0)=v(1)=0
\end{array}\right.
$$

where $f$ satisfies the following subcritical growth restriction (see [15], (6.1)):

$$
|f(s)| \leq C\left(1+|s|^{p}\right),
$$

for some $p \in\left(k, k^{*}\right]$. Here,

$$
k^{*}= \begin{cases}\frac{N(k+1)}{N-2 k}, & \text { if } k<\frac{N}{2}, \\ +\infty, & \text { if } k \geq \frac{N}{2} .\end{cases}
$$

These are the lower critical exponent for the $k$-Hessian operator (see $[26,27])$.
We assume that $f$ satisfies the following assumptions:
(H1) $s f(s)>0$ for $s \neq 0$
(H2) $f_{0}, f_{\infty} \in(0,+\infty)$
(H3) $f_{0}=\infty$ and $f_{\infty} \in(0, \infty)$
(H4) $f_{0} \in(0, \infty)$ and $f_{\infty}=\infty$
(H5) $f_{0}=0$ and $f_{\infty} \in(0, \infty)$.
(H6) $f_{0} \in(0, \infty)$ and $f_{\infty}=0$
(H7) $f_{0}=\infty$ and $f_{\infty}=0$
(H8) $f_{0}=0$ and $f_{\infty}=\infty$
(H9) $f_{0}=\infty$ and $f_{\infty}=\infty$
(H10) $f_{0}=0$ and $f_{\infty}=0$ 
Here,

$$
\begin{aligned}
f_{0} & =\lim _{|s| \longrightarrow 0} \frac{f(s)}{s^{k}}, \\
f_{\infty} & =\lim _{|s| \longrightarrow+\infty} \frac{f(s)}{s^{k}} .
\end{aligned}
$$

Obviously, any one-sign solution of problem (42) is a one-sign solution of problem (8). Conversely, we claim that any one-sign solution of problem (8) is a one-sign solution of problem (42).

Define the Nemytskii operator $H_{1}: \mathbb{R} \times E \longrightarrow Y$ by

$$
H_{1}(\mu, v)(r):=\frac{N}{C_{N}^{k}} r^{N-1}\left[\alpha(r)\left(v^{+}\right)^{k}+\beta(r)\left(v^{-}\right)^{k}+\mu a(r) f(v)\right] .
$$

Then, it is clear that $H_{1}$ is a continuous (compact) operator and problem (42) can be equivalently written as

$$
v=G_{k}\left[H_{1}(\mu, v)\right]:=F_{1}(\mu, v),
$$

where $F_{1}: \mathbb{R} \times E \longrightarrow E$ is completely continuous and $F_{1}(\mu, 0)=0, \forall \mu \in \mathbb{R}$.
The main results of this section are the following theorems.

Theorem 4. Let (AO), (A3), (H1), and (H2) hold. Then, for any $\gamma \in\left(\lambda^{\nu} / f_{\infty}, \lambda^{\nu} / f_{0}\right) \cup\left(\lambda^{\nu} / f_{0}, \lambda^{\nu} / f_{\infty}\right)$ with $\nu=+,-$, problem (8) possesses two solutions $u^{+}$and $u^{-}$such that $v u^{v}>0$.

Remark 4. From (H1) and (H2), we can see that there exists a positive constant $Q$ such that $f(s) / s \geq Q$ for all $s \neq 0$.

Remark 5. Note that if $\alpha(t)=\beta(t) \equiv 0$, the result of Theorem 4 is equivalent to those of Theorem 6.3 in [15]; Theorem 4 extends the corresponding results of [15].

In order to prove Theorem 4, similar to the proof of Lemma 2.3 of [15] and Lemma 3.1 of [17] with obvious change, we need the following Sturm-type comparison result.

Lemma 11. Let $b_{2}(r) \geq \max \left\{b_{1}(r), b_{1}(r)+\alpha+\beta, b_{1}(r)-\alpha-\right.$ $\beta\}$ for $r \in(0,1)$ and $b_{i}(r) \in C(0,1), i=1,2$. Also, let $v_{1}$ and $v_{2}$ be solutions of the following differential equations:

$$
\left\{\begin{array}{l}
\left(r^{N-k}\left(-v^{\prime}\right)^{k}\right)^{\prime}=\frac{N}{C_{N}^{k}} r^{N-1}\left[b_{i}(r) v^{k}+\alpha(r)\left(v^{+}\right)^{k}+\beta(r)\left(v^{-}\right)^{k}\right], \quad r \in(0,1), \\
v^{\prime}(0)=v(1)=0,
\end{array}\right.
$$

respectively. If $(c, d) \subseteq(0,1), v_{1}(c)=v_{1}(d)=0$, and $v_{1}(t) \neq 0$ in $(c, d)$, then either there exists $\tau \in(c, d)$ such that $v_{2}(\tau)=0$ or $b_{2}=b_{1}$ or $b_{2}=b_{1}+\alpha+\beta$ or $b_{2}=b_{1}-\alpha-\beta$ and $v_{2}(r)=$ $\mu v_{1}(r)$ for some constant $\mu \neq 0$.

Proof. If $u_{2}(r)=0$ on $(c, d)$, we discuss two cases.

Case 1. $u_{1}(r) u_{2}(r)>0$ in $(c, d)$, similar to the proof of Lemma 10. By Lemma 3 and 4 , we have that $u_{2}=\mu u_{1}$ and $b_{2}=b_{1}$.

Case 2. $u_{1}(r) u_{2}(r)<0$ in $(c, d)$, similar to the proof of Case 1. We have that $u_{2}=\mu u_{1}$ and $b_{2}=b_{1}+\alpha+\beta$ or $b_{2}=$ $b_{1}-\alpha-\beta$.

By Lemma 11, we obtain the following result that will be used later.

Lemma 12. Let $b_{i}(r) \in C(0,1), i=1,2 \quad$ and $\quad b_{2}(r) \geq$ $\max \left\{b_{1}(r), b_{1}(r)+\alpha+\beta, b_{1}(r)-\alpha-\beta\right\} \quad$ and $\quad b_{2}(r) \neq \equiv$ $\max \left\{b_{1}(r), b_{1}(r)+\alpha+\beta, b_{1}(r)-\alpha-\beta\right\}$ on $[0,1]$. Also let $v_{1}$ and $v_{2}$ be solutions of problem (48). If $v_{1}(r) \neq 0$ in $(0,1)$, then $v_{2}$ has at least one zero in $(0,1)$.
Proof of Theorem 4. It suffices to prove that problem (42) possesses two solutions $v^{+}$and $v^{-}$such that $v v^{\nu}>0$ for $v=+,-$. Note that $f_{0} \in(0,+\infty)$ implies $f(0)=0$. Hence, $v=0$ is always the solution of problem (42). Let $\zeta \in C(\mathbb{R}, \mathbb{R})$ such that $f(u)=f_{0} v^{k}+\zeta(v)$ with $\lim _{|s| \longrightarrow 0} \zeta(s) / s^{k}=0$. For $v=+,-$, applying Theorem 3 to problem (42), we know that there is an unbounded continua $\mathrm{D}^{\nu}$ of solutions to problem (42), bifurcating from $\left(\lambda^{\nu} / f_{0}, 0\right)$, such that

$$
D^{\nu} \subset\left(P^{v} \cup\left\{\left(\frac{\lambda^{\nu}}{f_{0}}, 0\right)\right\}\right) \text {. }
$$

To complete this theorem, it will be enough to show that $\mathrm{D}^{v}$ joins $\left(\lambda^{v} / f_{0}, 0\right)$ to $\left(\lambda^{\nu} / f_{\infty}, \infty\right)$. Let $\left(\mu_{n}, v_{n}\right) \in \mathrm{D}^{\nu} \backslash$ $\left\{\left(\lambda^{\nu} / f_{0}, 0\right)\right\}$ satisfy $\mu_{n}+\left\|v_{n}\right\| \longrightarrow \infty$. We note that $\mu_{n}>0$ for all $n \in \mathbb{N}$ since $(0,0)$ is the only solution of problem (42) for $\gamma=0$ and $\mathrm{D}^{\nu} \cap(\{0\} \times Y)=\varnothing$.

We divide the rest of proofs into two steps.

Step 1. We show that there exists a constant number $M>0$ such that $\mu_{n} \in(0, M]$, for all $n$.

On the contrary, we suppose that $\lim _{n \longrightarrow+\infty}\left|\mu_{n}\right|=+\infty$. Since $\left(\mu_{n}, v_{n}\right) \in \mathrm{D}^{v}$, it follows from the compactness of $G_{k}$ that 


$$
\left\{\begin{array}{l}
\left(r^{N-k}\left(-v_{n}^{\prime}\right)^{k}\right)^{\prime}=\frac{N}{C_{N}^{k}} r^{N-1}\left[\mu_{n} a(r) \widetilde{f_{n}}(r) v_{n}^{k}+\alpha(r)\left(v_{n}^{+}\right)^{k}+\beta(r)\left(v_{n}^{-}\right)^{k}\right], \quad r \in(0,1), \\
v^{\prime}(0)=v(1)=0,
\end{array}\right.
$$

where $\widetilde{f_{n}}:[0,1] \times[0, \infty)$ by

$$
\widetilde{f_{n}}(r)=\left\{\begin{array}{l}
\frac{f\left(v_{n}\right)}{v_{n}^{k}}, \quad v_{n} \neq 0, r \in[0,1] \\
f_{0}, \quad v_{n}=0, r \in[0,1]
\end{array}\right.
$$

By Remark 4, we have

$$
\lim _{n \longrightarrow+\infty} \mu_{n} \widetilde{f_{n}}(r)= \pm \infty
$$

Let $\psi^{\nu}$ be an eigenfunction corresponding to $\lambda^{\nu}$. But if $\lim _{n \longrightarrow+\infty} \mu_{n} \widetilde{f_{n}}(r)=-\infty$, applying Lemma 12 to $v_{n}$ and $\psi^{\nu}$, we have that $\psi^{\nu}$ must change sign for $n$ large enough, by $\psi^{\nu} \in P^{v}$, which is impossible. So $\lim _{n \longrightarrow+\infty}$ $\mu_{n} \widetilde{f_{n}}(r)=+\infty$, applying Lemma 12 to $\psi^{v}$ and $v_{n}$, we get that $v_{n}$ must change sign for $n$ large enough, and this contradicts the fact that $v_{n} \in P^{\nu}$.
Step 2. We show that $\mathrm{D}^{v}$ joins $\left(\lambda^{v} / f_{0}, 0\right)$ to $\left(\lambda^{v} / f_{\infty}, \infty\right)$. It follows from Step 1 that $\left\|v_{n}\right\| \longrightarrow+\infty$. Let $\xi \in C(\mathbb{R}, \mathbb{R})$ be such that $f(v)=f_{\infty} v^{k}+\xi(v)$. Then, $\lim _{v \longrightarrow+\infty} \xi(v) / v^{k}=0$. Let $\bar{\xi}(v)=\max \{|\xi(s)|: 0 \leq$ $|s| \leq v\}$, then $\bar{\xi}$ is nondecreasing. Set $\widetilde{\xi}(v)=\max \{|\xi(s)|$ : $v / 2 \leq|s| \leq v]\}$. Then, we have that

$$
\begin{aligned}
& \lim _{v \longrightarrow+\infty} \frac{\tilde{\xi}(v)}{v^{k}}=0, \\
& \bar{\xi}(v) \leq \bar{\xi}\left(\frac{v}{2}\right)+\tilde{\xi}(v) .
\end{aligned}
$$

It follows that

$$
\lim _{v \longrightarrow+\infty} \frac{\bar{\xi}(v)}{v^{k}}=0 .
$$

We divide the equation

$$
\left\{\begin{array}{l}
\left(r^{N-k}\left(-v_{n}^{\prime}\right)^{k}\right)^{\prime}=\frac{N}{C_{N}^{k}} r^{N-1}\left[\mu_{n} a(r) f_{\infty} v_{n}+\alpha(r)\left(v_{n}^{+}\right)^{k}+\beta(r)\left(v_{n}^{-}\right)^{k}+\mu_{n} a(r) \xi\left(v_{n}\right)\right], \quad r \in(0,1) \\
v_{n}(0)=v_{n}(1)=0
\end{array}\right.
$$

by $\left\|v_{n}\right\|^{k}$ and set $w_{n}=v_{n} /\left\|v_{n}\right\|$. Since $w_{n}$ is bounded in $C^{2}[0,1]$, choosing a subsequence and relabeling if necessary, we have that $w_{n} \longrightarrow w$ for some $w \in E$. Moreover, from (54) and the fact that $\bar{\xi}$ is nondecreasing, we have that

$$
\lim _{n \longrightarrow \infty} \frac{\left|\xi\left(v_{n}(t)\right)\right|}{\left\|v_{n}\right\|^{k}}=0, \quad \forall t \in[0,1],
$$

$$
\begin{aligned}
\frac{\left|\xi\left(v_{n}(r)\right)\right|}{\left\|v_{n}\right\|^{k}} \leq \frac{\bar{\xi}\left(\left|v_{n}(r)\right|\right)}{\left\|v_{n}\right\|^{k}} \leq \frac{\bar{\xi}\left(\left\|v_{n}(r)\right\|_{\infty}\right)}{\left\|v_{n}\right\|^{k}} \leq \frac{\bar{\xi}\left(\left\|v_{n}(r)\right\|\right)}{\left\|v_{n}\right\|^{k}} \longrightarrow 0, \\
n \longrightarrow \infty, \forall r \in[0,1] .
\end{aligned}
$$

since

$$
\left\{\begin{array}{l}
\left(r^{N-k}\left(-w_{n}^{\prime}\right)^{k}\right)^{\prime}=\frac{N}{C_{N}^{k}} r^{N-1}\left[\mu_{n} a(r) f_{\infty} w_{n}+\alpha(r)\left(w_{n}^{+}\right)^{k}+\beta(r)\left(w_{n}^{-}\right)^{k}\right], \quad r \in(0,1), \\
w_{n}^{\prime}(0)=w_{n}(1)=0,
\end{array}\right.
$$

$$
\mu=\frac{\lambda^{\nu}}{f_{\infty}} .
$$

Thus, $\mathrm{D}^{\nu}$ joins $\left(\lambda^{v} / f_{0}, 0\right)$ to $\left(\lambda^{v} / f_{\infty}, \infty\right)$. 
Remark 6. From the Proof of Theorem 4, we can see that if $f_{0}, f_{\infty} \in(0,+\infty)$, then there exist $\lambda_{2}>0$ and $\lambda_{3}>0$ such that problem (8) possesses two one-sign solutions $u^{+}$and $u^{-}\left(\nu u^{\nu}>0, v=+,-\right)$ for all $\lambda \in\left(-\lambda_{3},-\lambda_{2}\right) \cup\left(\lambda_{2}, \lambda_{3}\right)$ and has no one-sign solution for all $\lambda \in\left(-\lambda_{2}, 0\right) \cup\left(0, \lambda_{2}\right) \cup(-\infty$, $\left.-\lambda_{3}\right) \cup\left(\lambda_{3}, \infty\right)$.

Proof. We shall only prove the case of $\lambda \in\left(0, \lambda_{2}\right)$ since the proofs of the other cases are completely analogous.

Clearly, $f_{0}, f_{\infty} \in(0,+\infty)$ implies that there exists a positive constant $M$ such that

$$
\left|\frac{f(s)}{s^{k}}\right| \leq M, \quad \text { for any } s \neq 0 .
$$

It is sufficient to show that there exists $\lambda_{2}>0$ such that problem (42) has no solution for all $\lambda \in\left(0, \lambda_{2}\right)$. Suppose on the contrary that there exists one pair $(\mu, v) \in \mathrm{D}^{+}$such that $\mu \in\left(0,\left(\left(C_{N}^{k}-\alpha^{0}-\beta^{0}\right) /\left(\gamma a^{0} M\right)\right)\right)$. Let $w=v /\|v\|$. Obviously, one has that

$$
\begin{aligned}
1= & \|w\|=\| \int_{r}^{1}\left[\frac { N } { C _ { N } ^ { k } } s ^ { k - N } \int _ { 0 } ^ { s } \tau ^ { N - 1 } \left(\alpha(\tau)\left(w_{n}^{+}\right)^{k}\right.\right. \\
& \left.\left.+\beta(\tau)\left(w_{n}^{-}\right)^{k}+\mu \gamma a(\tau) \frac{f(v)}{\left\|v_{n}\right\|^{k}}\right) \mathrm{~d} \tau\right]^{1 / k} \mathrm{~d} s \| \\
\leq & \left(\frac{\alpha^{0}+\beta^{0}+\mu \gamma a^{0} M}{C_{N}^{k}}\right)^{1 / k}<1 .
\end{aligned}
$$

This is a contradiction.

From the Proof of Theorem 4 and Remark 6, we can deduce the following two corollaries.
Corollary 3. Assume that there exists a positive constant $\rho>0$ such that

$$
\frac{f(s)}{s^{k}} \geq \rho, \quad \text { for any } s \neq 0 .
$$

Then, there exists $\varsigma_{*}>0$ such that problem (8) has no onesign solution for any $\lambda \in\left(-\infty,-\varsigma_{*}\right) \cup\left(\varsigma_{*},+\infty\right)$.

Corollary 4. Assume that there exists a positive constant $\varrho>0$ such that

$$
\frac{f(s)}{s^{k}} \leq \varrho, \quad \text { for any } s \neq 0 .
$$

Then, there exists $\eta_{*}>0$ such that problem (8) has no onesign solution for any $\lambda \in\left(-\eta_{*}, 0\right) \cup\left(0, \eta_{*}\right)$.

Theorem 5. Let (AO), (A3), (H1), and (H3) hold. For $v \in\{+,-\}$, assume that one of the following conditions holds:

(i) $\gamma \in\left(0, \lambda^{\nu} / f_{\infty}\right)$, for $\lambda^{\nu}>0$

(ii) $\gamma \in\left(0, \lambda^{+} / f_{\infty}\right) \cup\left(\lambda^{-} / f_{\infty}, 0\right)$, for $\nu \lambda^{\nu}>0$

(iii) $\gamma \in\left(0, \lambda^{-} / f_{\infty}\right) \cup\left(\lambda^{+} / f_{\infty}, 0\right)$, for $v \lambda^{\nu}<0$

(iv) $\gamma \in\left(\lambda^{v} / f_{\infty}, 0\right)$, for $\lambda^{v}<0$

Then, problem (8) possesses two solutions $u^{+}$and $u^{-}$, such that $v u^{v}>0$ for $v=+,-$.

Proof. We shall only prove the case of (i) since the proofs of the cases for (ii), (iii), and (iv) are completely analogous.

Inspired by the idea of [28], we define the cutoff function of $f$ as follows:

$$
f^{[n]}(s):= \begin{cases}n s^{k}, & s \in\left[-\frac{1}{n}, \frac{1}{n}\right], \\ \left(f\left(\frac{2}{n}\right)-\frac{1}{n^{k-1}}\right) n s+\frac{2}{n^{k-1}}-f\left(\frac{2}{n}\right), & s \in\left(\frac{1}{n}, \frac{2}{n}\right), \\ -\left(f\left(-\frac{2}{n}\right)-\frac{(-1)^{k}}{n^{k-1}}\right) n s+\frac{2(-1)^{k}}{n^{k-1}}-f\left(-\frac{2}{n}\right), & s \in\left(-\frac{2}{n},-\frac{1}{n}\right), \\ f(s), & s \in\left[-\infty,-\frac{2}{n}\right] \cup\left[\frac{2}{n},+\infty\right] .\end{cases}
$$

We consider the following problem:

$$
\left\{\begin{array}{l}
\left(r^{N-k}\left(-v^{\prime}\right)^{k}\right)^{\prime}=\frac{N}{C_{N}^{k}} r^{N-1}\left[\alpha(r)\left(v^{+}\right)^{k}+\beta(r)\left(v^{-}\right)^{k}+\gamma a(r) f^{[n]}(v)\right], \quad r \in(0,1), \\
v^{\prime}(0)=v(1)=0 .
\end{array}\right.
$$


Clearly, we can see that $\lim _{n \longrightarrow+\infty} f^{[n]}(s)=f(s)$, $\left(f^{[n]}\right)_{0}=n$, and $\left(f^{[n]}\right)_{\infty}=f_{\infty}$.

Similar to the Proof of Theorem 4, there exists an unbounded continuum $\mathrm{D}^{\nu[n]}$ of solutions of problem (65) emanating from $\left(\lambda^{\nu} / n, 0\right)$ and joining to $\left(\lambda^{\nu} / f_{\infty}, \infty\right)$, such that $\quad \mathrm{D}^{\nu[n]} \subset\left(\left(\mathbb{R} \times P^{\nu}\right) \cup\left\{\left(\lambda^{\nu} / n, 0\right)\right\}\right)$ and $\left(\lambda^{v} / f_{\infty}\right.$, $\infty) \in \mathrm{D}^{v[n]}$.

Taking $z^{*}=(0,0)$, we easily obtain that $z^{*} \epsilon$ $\liminf _{n \longrightarrow+\infty} \mathrm{D}^{v[n]}$. So condition (i) in Lemma 6 is satisfied with $z^{*}=(0,0)$.

Since $F_{1}$ is completely continuous from $\mathbb{R} \times E \longrightarrow E$, we have that $\left(\cup_{n=1}^{+\infty} \mathrm{D}^{\nu[n]}\right) \cap \bar{B}_{R}$ is precompact, and accordingly (ii) in Lemma 6 holds.

Therefore, by Lemma $6, \mathrm{D}^{v}=\limsup _{n \rightarrow \infty} \mathrm{D}^{v[n]}$ is unbounded, closed, and connected such that $z^{*} \in \mathrm{D}^{\nu}$.

Clearly, $v$ is the solution of problem (42) for any $(\lambda, u) \in \mathscr{D}^{\nu}$. From the definition of superior limit (see [24, p. $7])$, we can easily see that $\mathrm{D}^{v} \subseteq \cup_{n=1}^{\infty} \mathrm{D}^{v[n]}$. So one has that $\mathrm{D}^{\nu} \subseteq\left(\left(\mathbb{R} \times P^{v}\right) \cup\{(0,0)\}\right)$.

We may claim that $z^{*}=(0,0)$ is the unique bifurcation point of $\mathrm{D}^{v}$.

Suppose on the contrary that there exists a sequence $\left(\lambda_{n}, v_{n}\right) \in \mathrm{D}^{v} /\{(0,0)\}=\limsup _{n \rightarrow \infty} \mathrm{D}^{v[n]} /\{(0,0)\}$ such that $\lim _{n \longrightarrow \infty} \lambda_{n}=\mu \neq 0$ and $\lim _{n \longrightarrow \infty} \nu=0$. Hence, for any $N_{0} \in \mathbb{N}$, there exists $n_{0} \geq N_{0}$ such that $\left(\lambda_{n}, v_{n}\right) \in \mathrm{D}^{\nu\left[n_{0}\right]}$. By
(54), it follows that $\lambda_{n_{0}}=\lambda^{\nu} / n_{0}$ for $n_{0} \geq N_{0}$. From the arbitrary of $N_{0}$, it implies that $n_{0} \longrightarrow \infty$, i.e., $\mu=0$, which contradicts the assumption of $\mu \neq 0$.

Similar to the method of the proof of Step 2 in Theorem 4 , we can obtain that $\left(\lambda^{\nu} / f_{\infty}, \infty\right) \in \mathrm{D}^{\nu}$.

Thus, $\mathrm{D}^{v}$ is an unbounded component of solutions of problem (42) emanating from $(0,0)$, such that $\mathrm{D}^{\nu} \mathrm{C}$ $\left(\left(\mathbb{R} \times P^{v}\right) \cup\{(0,0)\}\right)$, and $\mathrm{D}^{\nu}$ joins $(0,0)$ to $\left(\lambda^{v} / f_{\infty}, \infty\right)$.

Theorem 6. Let (AO), (A3), (H1), (H4), and (43) hold. For $v \in\{+,-\}$, assume that one of the following conditions holds:

(i) $\gamma \in\left(0, \lambda^{\nu} / f_{0}\right)$, for $\lambda^{\nu}>0$

(ii) $\gamma \in\left(0, \lambda^{+} / f_{0}\right) \cup\left(\lambda^{-} / f_{0}, 0\right)$, for $\nu \lambda^{\nu}>0$

(iii) $\gamma \in\left(0, \lambda^{-} / f_{0}\right) \cup\left(\lambda^{+} / f_{0}, 0\right)$, for $\nu \lambda^{\nu}<0$

(iv) $\gamma \in\left(\lambda^{\nu} / f_{0}, 0\right)$, for $\lambda^{\nu}<0$

Then problem (8) possesses two solutions $u^{+}$and $u^{-}$, such that $v u^{v}>0$ for $v=+,-$.

Proof. We shall only prove the case of (i) since the proofs of the cases for (ii), (iii), and (iv) are completely analogous.

If $(\lambda, v)$ is any nontrivial solution of problem (42), dividing problem (42) by $\|v\|^{2 k}$ and setting $w=v /\|v\|^{2}$ yields

$$
\left\{\begin{array}{l}
\left(r^{N-k}\left(-w^{\prime}\right)^{k}\right)^{\prime}=\frac{N}{C_{N}^{k}} r^{N-1}\left[\alpha(r)\left(w^{+}\right)^{k}+\beta(r)\left(w^{-}\right)^{k}+\gamma a(r) \frac{f(v)}{\|v\|^{2 k}}\right], \quad r \in(0,1), \\
w^{\prime}(0)=w(1)=0
\end{array}\right.
$$

Define

Evidently, problem (66) is equivalent to

$$
\tilde{f}(w):= \begin{cases}\|w\|^{2 k} f\left(\frac{w}{\|w\|^{2}}\right), & \text { if } w \neq 0 \\ 0, & \text { if } w=0 .\end{cases}
$$

$$
\left\{\begin{array}{l}
\left(r^{N-k}\left(-w^{\prime}\right)^{k}\right)^{\prime}=\frac{N}{C_{N}^{k}} r^{N-1}\left[\alpha(r)\left(w^{+}\right)^{k}+\beta(r)\left(w^{-}\right)^{k}+\gamma a(r) \tilde{f}(w)\right], \quad r \in(0,1), \\
w^{\prime}(0)=w(1)=0
\end{array}\right.
$$

It is obvious that $(\gamma, 0)$ is always the solution of problem (68). By simple computation, we can show that $\widetilde{f}_{0}=f_{\infty}=$ $\infty$ and $\widetilde{f}_{\infty}=f_{0} \in(0, \infty)$. Now, applying Theorem 5 , there exists an unbounded continuum $\mathrm{C}^{\nu}$ of solutions of problem (68) emanating from $(0,0)$, such that $C^{\nu} \subset\left(\left(\mathbb{R} \times P^{\nu}\right) \cup\right.$ $\{(0,0)\})$, and $\mathrm{D}^{\nu}$ joins $(0,0)$ to $\left(\lambda^{\nu} / \widetilde{f}_{\infty}, \infty\right)$.

Under the inversion $w \longrightarrow w /\|w\|^{2}=v$, we obtain $\mathrm{C}^{v} \longrightarrow \mathrm{D}^{v}$ being an unbounded component of solutions of problem (42) emanating from $\left(\lambda^{v} / f_{0}, 0\right)$. Applying a similar method as the proof of Theorem 6.5 in [15], by (43), we can get that $(0, \infty) \in \mathrm{D}^{v}$.

Theorem 7. Let (AO), (A3), (H1), and (H5) hold. For $v \in\{+,-\}$, assume that one of the following conditions holds:

(i) $\gamma \in\left(\lambda^{\nu} / f_{\infty},+\infty\right)$, for $\lambda^{v}>0$

(ii) $\gamma \in\left(\lambda^{+} / f_{\infty},+\infty\right) \cup\left(-\infty, \lambda^{-} / f_{\infty}\right)$, for $\nu \lambda^{\nu}>0$ 
(iii) $\gamma \in\left(\lambda^{-} / f_{\infty},+\infty\right) \cup\left(-\infty, \lambda^{+} / f_{\infty}\right)$, for $\nu \lambda^{\nu}<0$

(iv) $\gamma \in\left(-\infty, \lambda^{v} / f_{\infty}\right)$, for $\lambda^{v}<0$

Then, problem (8) possesses two solutions $u^{+}$and $u^{-}$, such that $v u^{v}>0$ for $v=+,-$.
Proof. We shall only prove the case of (i) since the proofs of the cases for (ii), (iii), and (iv) are completely analogous.

Define

$$
f^{[n]}(s):= \begin{cases}\frac{1}{n} s^{k}, & s \in\left[-\frac{1}{n}, \frac{1}{n}\right], \\ {\left[f\left(\frac{2}{n}\right)-\frac{1}{n^{k+1}}\right] n s+\frac{2}{n^{k+1}}-f\left(\frac{2}{n}\right),} & s \in\left(\frac{1}{n}, \frac{2}{n}\right), \\ -\left[f\left(-\frac{2}{n}\right)-\frac{(-1)^{k}}{n^{k+1}}\right] n s+\frac{2(-1)^{k}}{n^{k+1}}-f\left(-\frac{2}{n}\right), & s \in\left(-\frac{2}{n},-\frac{1}{n}\right), \\ f(s), & s \in\left(-\infty,-\frac{2}{n}\right] \cup\left[\frac{2}{n},+\infty\right) .\end{cases}
$$

We consider the following problem:

$$
\left\{\begin{array}{l}
\left(r^{N-k}\left(-v^{\prime}\right)^{k}\right)^{\prime}=\frac{N}{C_{N}^{k}} r^{N-1}\left[\alpha(r)\left(v^{+}\right)^{k}+\beta(r)\left(v^{-}\right)^{k}+\gamma a(r) f^{[n]}(v)\right], \quad r \in(0,1), \\
v^{\prime}(0)=v(1)=0 .
\end{array}\right.
$$

Clearly, we can see that $\lim _{n \longrightarrow+\infty} f^{[n]}(s)=f(s)$, $\left(f^{[n]}\right)_{0}=1 / n$, and $\left(f^{[n]}\right)_{\infty}=f_{\infty}$.

Similar to the Proof of Theorem 4, there exists an unbounded continuum $\mathrm{D}^{\nu[n]}$ of solutions of problem (70) emanating from $\left(\lambda^{v} n, 0\right)$ and joining to $\left(\lambda^{v} / f_{\infty}, \infty\right)$, such that $\mathrm{D}^{\nu[n]} \subset\left(\left(\mathbb{R} \times P^{v}\right) \cup\left\{\left(\lambda^{v} n, 0\right)\right\}\right)$ and $\left(\lambda^{v} / f_{\infty}, \infty\right) \in \mathrm{D}^{\nu[n]}$.

Taking $z^{*}=(\infty, 0)$, we easily obtain that $z^{*} \in \liminf _{n \longrightarrow+\infty} \mathrm{D}^{\nu[n]}$ with $\left\|z^{*}\right\|_{\mathbb{R} \times E}=+\infty$ So condition (i) in Lemma 7 is satisfied with $z^{*}=(+\infty, 0)$.

Define a mapping $T: R \times E \longrightarrow R \times E$ such that

$$
T(\gamma, v)= \begin{cases}\left(\frac{1}{\gamma}, v\right), & \gamma \in(-\infty, 0) \cup(0,+\infty), \\ (0, v), & \gamma=\infty, \\ (\infty, v), & \gamma=0 .\end{cases}
$$

It is easy to verify that $T$ is a homeomorphism and $\left\|T\left(z^{*}\right)\right\|_{\mathbb{R} \times E}=0$. Obviously, $\left\{T\left(\mathrm{D}^{\nu[n]}\right)\right\}$ be a sequence of unbounded connected subsets in $E$. So (ii) in Lemma 7 holds.

Since $F_{1}$ is completely continuous from $\mathbb{R} \times E \longrightarrow E$, we have that $\left(\cup_{n=1}^{+\infty} T\left(\mathrm{D}^{\nu[n]}\right)\right) \cap \bar{B}_{R}$ is $s$ precompact, and accordingly (iii) in Lemma 7 holds.

Therefore, by Lemma $7, \mathrm{D}^{v}=\limsup _{n \rightarrow \infty} \mathrm{D}^{\nu[n]}$ is unbounded, closed, and connected such that $z^{*} \in \mathrm{D}^{\nu}$.
Obviously, $v$ is the solution of problem (42) for any $(\gamma, v) \in \mathrm{D}^{\nu}$. From the definition of superior limit (see [24], P. 7), we can easily see that $\mathrm{D}^{v} \subseteq \cup_{n=1}^{\infty} \mathrm{D}^{v[n]}$. So one has that $\mathrm{D}^{v} \subset\left(\mathbb{R} \times\left(P^{v} \cup\{0\}\right)\right)$.

Next, we show that the projection of $\mathrm{D}^{\nu}$ on $\mathbb{R}$ is nonempty. By Theorem 4, we have known that $\mathrm{D}^{v[n]}$ has unbounded projection on $\mathbb{R}$ for any fixed $n \in \mathbb{N}$. By Lemma 8 , for each fixed $\epsilon>0$, there exists an $m$ such that for every $n>m, \mathrm{D}^{v[n]} \subset V_{\epsilon}\left(\mathrm{D}^{\nu}\right)$. This implies that

$$
\left(\lambda^{v} n, \infty\right) \subseteq \operatorname{Proj}_{\mathbb{R}}\left(\mathrm{D}^{\nu[n]}\right) \subseteq \operatorname{Proj}_{\mathbb{R}}\left(V_{\epsilon}\left(\mathrm{D}^{\nu}\right)\right),
$$

where $\operatorname{Proj}_{\mathbb{R}}\left(\mathrm{D}^{\nu}\right)$ denotes the projection of $\mathrm{D}^{\nu}$ on $\mathbb{R}$. It follows that the projection of $\mathrm{D}^{v}$ is nonempty on $\mathbb{R}$.

Theorem 8. Let (A0), (A3), (H1), and (H6) hold. For $v \in\{+,-\}$, assume that one of the following conditions holds:

(i) $\gamma \in\left(\lambda^{v} / f_{0},+\infty\right)$, for $\lambda^{v}>0$

(ii) $\gamma \in\left(-\infty, \lambda^{+} / f_{0}\right) \cup\left(\lambda^{-} / f_{0}\right),+\infty$, for $\nu \lambda^{\nu}>0$

(iii) $\gamma \in\left(-\infty, \lambda^{-} / f_{0}\right) \cup\left(\lambda^{+} / f_{0},+\infty\right)$, for $\nu \lambda^{\nu}<0$

(iv) $\gamma \in\left(-\infty, \lambda^{v} / f_{0}\right)$, for $\lambda^{\nu}<0$

Then, problem (8) possesses two solutions $u^{+}$and $u^{-}$, such that $v u^{\nu}>0$ for $v=+,-$. 
Proof. Applying a similar method as the proof of Theorem 6 and the conclusion of Theorem 7 , we can easily get the desired conclusion.

Theorem 9. Let (AO), (A3), (H1), and (H7) hold. For $v \in\{+,-\}$, assume that one of the following conditions holds:

(i) $\gamma \in(0,+\infty)$, for $\lambda^{v}>0$ (ii) or $\gamma \in(0,+\infty) \cup(-\infty, 0)$, for $\nu \lambda^{\nu}>0$, or $\nu \lambda^{\nu}<0$

(iii) $\gamma \in(-\infty, 0)$, for $\lambda^{\nu}<0$

Then, problem (8) possesses two solutions $u^{+}$and $u^{-}$, such that $v u^{v}>0$ for $v=+,-$.

Define

$$
f^{[n]}(s):= \begin{cases}\frac{1}{n} s^{k}, & s \in(-\infty,-2 n] \cup[2 n,+\infty) . \\ \frac{-(-2)^{k} n^{k-1}+f(-n)}{n}(s+n)+f(-n), & s \in(-2 n,-n), \\ \frac{2^{k} n^{k-1}-f(n)}{n}(s-n)+f(n), & s \in\left[-n,-\frac{2}{n}\right] \cup\left[\frac{2}{n}, n\right], \\ -\left[f\left(-\frac{2}{n}\right)-\frac{(-1)^{k}}{n^{k-1}}\right] n s+\frac{2(-1)^{k}}{n^{k-1}}-f\left(-\frac{2}{n}\right), & s \in\left(-\frac{2}{n},-\frac{1}{n}\right), \\ {\left[f\left(\frac{2}{n}\right)-\frac{1}{n^{k-1}}\right] n s+\frac{2}{n^{k-1}}-f\left(\frac{2}{n}\right),} & s \in\left(\frac{1}{n}, \frac{2}{n}\right), \\ n s^{k}, & s \in\left[-\frac{1}{n}, \frac{1}{n}\right] .\end{cases}
$$

We consider the following problem

$$
\left\{\begin{array}{l}
\left(r^{N-k}\left(-v^{\prime}\right)^{k}\right)^{\prime}=\frac{N}{C_{N}^{k}} r^{N-1}\left[\alpha(r)\left(v^{+}\right)^{k}+\beta(r)\left(v^{-}\right)^{k}+\gamma a(r) f^{[n]}(v)\right], \quad r \in(0,1), \\
v^{\prime}(0)=v(1)=0 .
\end{array}\right.
$$

Clearly, we can see that $\lim _{n \longrightarrow+\infty} f^{[n]}(s)=f(s)$, $\left(f^{[n]}\right)_{0}=n$, and $\left(f^{[n]}\right)_{\infty}=1 / n$.

Similar to the Proof of Theorem 4, there exists an unbounded continuum $\mathrm{D}^{v[n]}$ of solutions of problem (74) emanating from $\left(\lambda^{v} / n, 0\right)$, such that $\mathrm{D}^{\nu[n]} \subset\left(\left(\mathbb{R} \times P^{v}\right) \cup\right.$ $\left.\left\{\left(\lambda^{\nu} / n, 0\right)\right\}\right)$ and $\left(\lambda^{\nu} n, \infty\right) \in \mathrm{D}^{\nu[n]}$.

Taking $z_{n}=\left(\lambda^{v} / n, 0\right)$ and $z^{*}=(0,0)$, we have that $z_{n} \longrightarrow z^{*}$. Similar to the proof of Theorem 5 , by Lemma 6 , $\mathrm{D}^{v}=\lim \sup _{n \longrightarrow \infty} \mathrm{D}^{v[n]}$ is unbounded, closed, and connected such that $z^{*}=(0,0) \in \mathrm{D}^{v}$, and $z^{*}=(0,0)$ is the unique bifurcation point of $\mathrm{D}^{\nu}$. By Theorem 7 , we have $z^{*}=(\infty, \infty) \in \mathrm{D}^{\nu}$.

Thus, we can obtain the results.
Theorem 10. Let (A0), (A3), (H1), (H8), and (43) hold. For $\nu \in\{+,-\}$, assume that one of the following conditions holds:

(i) $\gamma \in(0,+\infty)$, for $\lambda^{\nu}>0$

(ii) or $\gamma \in(0,+\infty) \cup(-\infty, 0)$, for $\nu \lambda^{\nu}>0$, or $\nu \lambda^{\nu}<0$

(iii) $\gamma \in(-\infty, 0)$, for $\lambda^{\nu}<0$

Then, problem (8) possesses two solutions $u^{+}$and $u^{-}$, such that $v u^{v}>0$ for $v=+,-$.

Proof. Applying a similar method as the proof of Theorem 6 and the conclusion of Theorem 9, we can easily get the desired conclusion. 
Theorem 11. Let (A0), (A3), (H1), (H9), and (43) hold. For $v \in\{+,-\}$, assume that one of the following conditions holds:

(i) There exists a $\lambda_{\nu}^{v}>0$ for $\lambda^{v}>0$, such that $\gamma \in\left(0, \lambda_{\nu}^{v}\right)$

(ii) There exists a $v \lambda_{\nu}^{\nu}>0$ for $\nu \lambda^{v}>0$, such that $\gamma \in$ $\left(\lambda_{-}^{-}, 0\right) \cup\left(0, \lambda_{+}^{+}\right)$

(iii) There exists a $v \lambda_{\nu}^{\nu}<0$ for $\nu \lambda^{\nu}<0$, such that, $\gamma \in$ $\left(\lambda_{+}^{+}, 0\right) \cup\left(0, \lambda_{-}^{-}\right)$ (iv) There exists a $\lambda_{\nu}^{v}<0$ for $\lambda^{\nu}<0$, such that $\gamma \in\left(\lambda_{\nu}^{v}, 0\right)$

Then, problem (8) possesses two solutions $u^{+}$and $u^{-}$, such that $\nu u^{v}>0$ for $v=+,-$.

Proof. Define

$$
f^{[n]}(s):= \begin{cases}n s^{k}, & s \in(-\infty,-2 n] \cup[2 n,+\infty) . \\ \frac{-(-2)^{k} n^{k+1}+f(-n)}{n}(s+n)+f(-n), & s \in(-2 n,-n), \\ \frac{2^{k} n^{k+1}-f(n)}{n}(s-n)+f(n), & s \in(n, 2 n), \\ -\left[f\left(-\frac{2}{n}\right)-\frac{(-1)^{k}}{n^{k-1}}\right] n s+\frac{2(-1)^{k}}{n^{k-1}}-f\left(-\frac{2}{n}\right), & s \in\left(-\frac{2}{n},-\frac{1}{n}\right) \\ {\left[f\left(\frac{2}{n}\right)-\frac{1}{n^{k-1}}\right] n s+\frac{2}{n^{k-1}}-f\left(\frac{2}{n}\right),} & s \in\left(\frac{1}{n}, \frac{2}{n}\right) \\ n s^{k}, & s \in\left[-\frac{1}{n}, \frac{1}{n}\right] .\end{cases}
$$

We consider the following problem:

$$
\left\{\begin{array}{l}
\left(r^{N-k}\left(-v^{\prime}\right)^{k}\right)^{\prime}=\frac{N}{C_{N}^{k}} r^{N-1}\left[\alpha(r)\left(v^{+}\right)^{k}+\beta(r)\left(v^{-}\right)^{k}+\gamma a(r) f^{[n]}(v)\right], \quad r \in(0,1), \\
v^{\prime}(0)=v(1)=0 .
\end{array}\right.
$$

Clearly, we can see that $\lim _{n \longrightarrow+\infty} f^{[n]}(s)=f(s)$, $\left(f^{[n]}\right)_{0}=n$ and $\left(f^{[n]}\right)_{\infty}=n$.

Similar to the Proof of Theorem 4, there exists an unbounded continuum $\mathrm{D}^{\nu[n]}$ of solutions of problem (48) emanating from $\left(\lambda^{\nu} / n, 0\right)$, such that $\mathrm{D}^{\gamma[n]} \subset\left(\left(\mathbb{R} \times P^{\nu}\right) \cup\right.$ $\left.\left\{\left(\lambda^{\nu} / n, 0\right)\right\}\right)$ and $\left(\lambda^{\nu} / n,+\infty\right) \in \mathrm{D}^{\nu[n]}$.

Taking $z_{n}=\left(\lambda^{\nu} / n, 0\right)$ and $z^{*}=(0,0)$, we have that $z_{n} \longrightarrow z^{*}$. Similar to the proof of Theorem 5 , by Lemma 6 , $\mathrm{D}^{v}=\limsup _{n \rightarrow \infty} \mathrm{D}^{v[n]}$ is unbounded, closed, and connected such that $(0,0) \in \mathrm{D}^{\nu}$. By Theorem 6 , we have that $(0, \infty) \in \mathrm{D}^{\nu}$.

Thus, we can easily get the results of this theorem.

Theorem 12. Let (AO), (A3), (H1), and (H10) hold. For $v \in\{+,-\}$, assume that one of the following conditions holds: (i) There exists a $\lambda_{\nu}^{\nu}>0$ for $\lambda^{\nu}>0$, such that $\gamma \in\left(\lambda_{\nu}^{\nu},+\infty\right)$

(ii) There exists a $\nu \lambda_{\nu}^{\nu}>0$ for $\nu \lambda^{\nu}>0$, such that $\gamma \in$ $\left(-\infty, \lambda_{-}^{-}\right) \cup\left(\lambda_{+}^{+},+\infty\right)$

(iii) There exists a $\nu \lambda_{\nu}^{v}<0$ for $\nu \lambda^{v}<0$, such that $\gamma \in$ $\left(-\infty, \lambda_{+}^{+}\right) \cup\left(\lambda_{-}^{-},+\infty\right)$

(iv) There exists a $\lambda_{\nu}^{v}<0$ for $\lambda^{v}<0$, such that $\gamma \in\left(-\infty, \lambda_{\gamma}^{v}\right)$

Then, problem (8) possesses two solutions $u^{+}$and $u^{-}$, such that $\nu \mathcal{u}^{v}>0$ for $v=+,-$.

Proof. Using an argument similar to that of Theorem 6, in view of Theorem 11, we can easily get the results of this theorem. 


\section{Data Availability}

Data sharing not applicable to this paper as no datasets were generated or analyzed during the current study.

\section{Conflicts of Interest}

The authors declare that they have no conflicts of interest.

\section{Authors' Contributions}

The author conceived the study, drafted the manuscript, and approved the final manuscript.

\section{Acknowledgments}

This research was supported by the NSFC (no. 11561038) and the "Kaiwu"Innovation Team Support Project of Lanzhou Institute of Technology (no.2018KW-03).

\section{References}

[1] K.-S. Chou, D. Geng, and S.-S. Yan, "Critical dimension of a Hessian equation involving critical exponent and a related asymptotic result," Journal of Differential Equations, vol. 129, no. 1, pp. 79-110, 1996.

[2] K.-S. Chou and X.-J. Wang, "A variational theory of the Hessian equation," Communications on Pure and Applied Mathematics, vol. 54, no. 9, pp. 1029-1064, 2001.

[3] H. Dong, "Hessian equations with elementary symmetric functions," Communications in Partial Differential Equations, vol. 31, no. 7, pp. 1005-1025, 2006.

[4] B. Guan, "The Dirichlet problem for Hessian equations on Riemannian manifolds," Calculus of Variations and Partial Differential Equations, vol. 8, no. 1, pp. 45-69, 1999.

[5] N. Ivochkina, N. Trudinger, and X.-J. Wang, "The Dirichlet problem for degenerate Hessian equations," Communications in Partial Differential Equations, vol. 29, no. 1-2, pp. 219-235, 2005.

[6] H. Jian, "Hessian equations with infinite Dirichlet boundary," Indiana University Mathematics Journal, vol. 55, no. 3, pp. 1045-1062, 2006.

[7] D. A. Labutin, "Potential estimates for a class of fully nonlinear elliptic equations," Duke Mathematical Journal, vol. 111, no. 1, pp. 1-49, 2002.

[8] R. C. Reilly, "On the Hessian of a function and the curvatures of its graph," The Michigan Mathematical Journal, vol. 20, no. 1, pp. 373-383, 1974.

[9] W.-M. Sheng, N. S. Trudinger, and X.-J. Wang, "The Yamabe problem for higher order curvatures," Journal of Differential Geometry, vol. 77, no. 3, pp. 515-553, 2007.

[10] N. S. Trudinger and X.-J. Wang, "A Poincaré type inequality for Hessian integrals," Calculus of Variations and Partial Differential Equations, vol. 6, no. 4, pp. 315-328, 1998.

[11] N. S. Trudinger and X.-J. Wang, "Hessian measures III," Journal of Functional Analysis, vol. 193, no. 1, pp. 1-23, 2002.

[12] K. Tso, "On symmetrization and Hessian equations," Journal d'Analyse Mathématique, vol. 52, no. 1, pp. 94-106, 1981.

[13] M. Warren and Y. Yuan, "Hessian estimates for the sigma-2 equation in dimension 3," Communications on Pure and Applied Mathematics, vol. 62, no. 3, pp. 305-321, 2009.
[14] X.-J. Wang, "A class of fully nonlinear elliptic equations and related functionals," Indiana University Mathematics Journal, vol. 43, no. 1, pp. 25-54, 1994.

[15] G. Dai, "Bifurcation and admissible solutions for the Hessian equation," Journal of Functional Analysis, vol. 273, no. 10, pp. 3200-3240, 2017.

[16] H. Berestycki, "On some nonlinear Sturm-Liouville problems," Journal of Differential Equations, vol. 26, no. 3, pp. 375-390, 1977.

[17] G. Dai and R. Ma, "Unilateral global bifurcation for $p$-Laplacian with non- $p-1$-linearization nonlinearity," Discrete \& Continuous Dynamical Systems-A, vol. 35, no. 1, pp. 99-116, 2015.

[18] G. Dai and R. Ma, "Global bifurcation, Berestycki's conjecture and one-sign solutions for $p$-Laplacian," Nonlinear Analysis: Theory, Methods and Applications, vol. 91, pp. 51-59, 2013.

[19] W. Shen, "Unilateral global interval bifurcation for Kirchhoff type problems and its applications," Communications on Pure and Applied Analysis, vol. 17, no. 1, pp. 21-37, 2018.

[20] W. Shen, "Global interval bifurcation and convex solutions for the Monge-Ampre equations," Electronic Journal of Differential Equations, vol. 2018, no. 2, pp. 1-15, 2018.

[21] K. Tso, "Remarks on critical exponents for Hessian operators," Annales de l'Institut Henri Poincare (C) Non Linear Analysis, vol. 7, no. 2, pp. 113-122, 1990.

[22] P. H. Rabinowitz, "Some global results for nonlinear eigenvalue problems," Journal of Functional Analysis, vol. 7, no. 3, pp. 487-513, 1971.

[23] E. Dancer, "On the structure of solutions of non-linear eigenvalue problems," Indiana University Mathematics Journal, vol. 23, no. 11, pp. 1069-1076, 1974.

[24] G. T. Whyburn, Topological Analysis, p.7, Princeton University Press, Princeton, NJ, USA, 1958.

[25] G. Dai, "Bifurcation and one-sign solutions of the $p$-Laplacian involving a nonlinearity with zeros," Discrete and Continuous Dynamical Systems, vol. 36, no. 10, pp. 5323-5345, 2016.

[26] N. C. Phuc and I. E. Verbitsky, "Local integral estimates and removable singularities for quasilinear and Hessian equations with nonlinear source terms," Communications in Partial Differential Equations, vol. 31, no. 12, pp. 1779-1791, 2006.

[27] N. Phuc and I. Verbitsky, "Quasilinear and hessian equations of Lane-Emden type," Annals of Mathematics, vol. 168, no. 3, pp. 859-914, 2008.

[28] A. Ambrosetti, R. M. Calahorrano, and F. R. Dobarro, "Global branching for discontinuous problems," Commentationes Mathematicae Universitatis Carolinae, vol. 31, pp. 213-222, 1990. 